\title{
Filigrane
}

Écoutes psychanalytiques

\section{Comment comprendre la violence des adolescents délinquants}

\section{Maurice Berger}

Volume 26, numéro 1, 2017

La terreur des enfants : première partie

URI : https://id.erudit.org/iderudit/1041689ar

DOI : https://doi.org/10.7202/1041689ar

Aller au sommaire du numéro

Éditeur(s)

Revue Santé mentale au Québec

ISSN

1192-1412 (imprimé)

1911-4656 (numérique)

Découvrir la revue

Citer cet article

Berger, M. (2017). Comment comprendre la violence des adolescents délinquants. Filigrane, 26(1), 13-39. https://doi.org/10.7202/1041689ar

\section{Résumé de l'article}

À partir d'observations réalisées lors demultiples interventions à titre de pédopsychiatred'orientation psychanalytique dans un Centre éducatifrenforcé en France, cet article se propose d'analysercertains processus psychiques et identitaires en jeu chez lesadolescents. Nous décrirons plus particulièrement lesdiverses composantes de la joute qui a souvent lieu dans le rapport duclinicien aux adolescents, en particulier avec ceux issus de milieuximmigrés et ayant commis des délits violents. Il sembleau premier abord difficile de transposer l'expériencethérapeutique usuelle avec de tels adolescents, ce qui impliqueune attention particulière à leur histoire, qui comportegénéralement des traumatismes relationnelsprécoces, notamment de négligence et de violence. Oncomprend alors comment pour de tels jeunes, l'extérieurpuisse être plus protecteur que l'intérieur. Enconséquence, pour faire advenir de la pensée chez eux, ilfaut savoir les sécuriser et les contenir en reconnaissant leurméfiance, et surtout, à la lumière des travaux deWinnicott et de Bion, savoir susciter une curiosité et uneco-créativité.
Ce document est protégé par la loi sur le droit d'auteur. L'utilisation des services d’Érudit (y compris la reproduction) est assujettie à sa politique d'utilisation que vous pouvez consulter en ligne.

https://apropos.erudit.org/fr/usagers/politique-dutilisation/ 


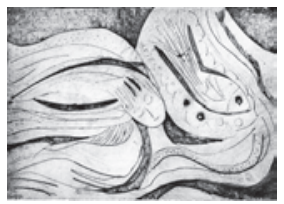

\title{
Comment comprendre la violence des adolescents délinquants
}

\author{
Maurice Berger
}

RÉSUMÉ : À partir d'observations réalisées lors de multiples interventions à titre de pédopsychiatre d'orientation psychanalytique dans un Centre éducatif renforcé en France, cet article se propose d'analyser certains processus psychiques et identitaires en jeu chez les adolescents. Nous décrirons plus particulièrement les diverses composantes de la joute qui a souvent lieu dans le rapport du clinicien aux adolescents, en particulier avec ceux issus de milieux immigrés et ayant commis des délits violents. Il semble au premier abord difficile de transposer l'expérience thérapeutique usuelle avec de tels adolescents, ce qui implique une attention particulière à leur histoire, qui comporte généralement des traumatismes relationnels précoces, notamment de négligence et de violence. On comprend alors comment pour de tels jeunes, l'extérieur puisse être plus protecteur que l'intérieur. En conséquence, pour faire advenir de la pensée chez eux, il faut savoir les sécuriser et les contenir en reconnaissant leur méfiance, et surtout, à la lumière des travaux de Winnicott et de Bion, savoir susciter une curiosité et une co-créativité.

Mots clés: Psychanalyse de l'adolescence, violence, traumatisme, négligence, destructivité.

\begin{abstract}
Based on multiple interventions as a psychoanalytic psychiatrist in a reinforced educational center in France, this article aims to analyze certain psychic and identity issues that are at play in adolescents. More specifically, we will describe the various components of the verbal jousting that often occurs in the relationship between clinicians and adolescents, particularly when they come from immigrant backgrounds and have already committed violent offenses. At first glance, it seems indeed difficult to translate the ordinary therapeutic experience with such adolescents. The clinician must pay close attention to their history, which usually includes early relational trauma, neglect and violence. Hence, for such teenagers, the outside appears more protective than the interior. In order to make room for thought and elaboration, it is necessary to make them feel secure by recognizing their initial mistrust, and, in the light of the works of Winnicott and Bion, by inciting curiosity and co-creativity.
\end{abstract}

Keywords: Adolescent Psychoanalysis, violence, trauma, neglect, destructivity. 


\section{Introduction}

es pistes de réflexion qui suivent ne prétendent pas saisir la totalité des — processus en jeu chez les adolescents violents et délinquants. Elles sont issues de mon intervention, comme pédopsychiatre de formation psychanalytique, dans un Centre éducatif renforcé (CER), et leur apport est modeste comparé à l'action quotidienne de l'équipe éducative. Ma motivation pour effectuer ce travail est de tenter de diminuer le nombre de victimes que font répétitivement ces jeunes.

J'ai remarqué que la veille d'un entretien avec ces adolescents, je fais attention d'être bien en forme physiquement et psychiquement pour le lendemain. Dans un premier temps, j'ai pensé que ceci était dû au fait que, ces jeunes ayant de grandes difficultés de mentalisation, je devais être particulièrement attentif à saisir la moindre occasion de leur permettre de penser sur eux-mêmes. Je devais presque penser pour deux. Cette hypothèse n'était pas fausse, mais insuffisante.

De leur côté, dans la voiture qui les amenait à notre rendez-vous, la plupart de ces sujets expliquaient à leur éducateur que le futur entretien avec moi leur «cassait les couilles» et qu'ils allaient en partir au bout de cinq minutes. Il m'a fallu plus d'un an pour réaliser que ces sujets injectaient une tension très importante dans ces entretiens et que, sans me l'être formulé, j'entrais avec eux dans une joute verbale dont je décrirai plus loin les composantes, d'où la nécessité d'être en forme.

Un autre élément intriguant me permit d'avancer dans la compréhension des processus en jeu. Un jeune particulièrement tendu, au point d'être debout à un moment de la discussion, et qui m'avait dit avoir «un caractère d'enculé» - à traduire par «un très mauvais caractère»-, demanda quelques jours plus tard à me revoir. Il voulait ce nouvel entretien car, selon son expression, «le Dr Berger, c'est lui qui est bizarre». Un aspect bizarre qui ne l'inquiétait pas puisqu'il souhaitait revenir. Donc il était devenu un peu curieux. Je mis cela dans un coin de ma tête. Peu après, un autre adolescent rouspétait sans arrêt contre mes questions et ne me laissait d'ailleurs guère le temps d'en poser; il refusait toute réflexion à leur sujet et, très vindicatif, il me déclara que «parler du passé ne sert à rien, les gens comme vous grattent là où ça fait mal, pourquoi faites-vous cela?» Je lui répondis que c'est parce que je veux essayer de le transformer en chat. On remarque l'alternance sensorielle «je veux» dur, «essayer» souple, «te transformer» dur, «chat» souple. Intrigué, il m’interroge, et je lui explique que les chats sont curieux, ils veulent toujours aller voir dans les endroits qu'ils ne connaissent pas, ce 
qui leur joue parfois des tours. Et j'aimerais qu'il devienne curieux à l'égard de lui-même. La discussion se poursuit alors sur un ton plus calme, car la réflexion commune a une dimension moins intrusive.

Accepter tranquillement la joute et faire preuve d'une curiosité partagée à égalité permet que nos entretiens durent non pas cinq minutes, mais entre trois-quarts d'heure et une heure, et presque tous ces adolescents acceptent un autre rendez-vous.

\section{Le cadre de ce travail}

\section{Un CER assez contenant}

Les deux responsables institutionnels sont engagés et l'ambiance institutionnelle permet la pensée. Les prises en charge actuelles sont incluses dans un dispositif de recherche expérimental et s'accompagnent de bilans psychomoteurs et pédagogiques spécifiques. Par ailleurs, les adolescents ont des entretiens réguliers dans l'institution avec des psychologues, entretiens plus ciblés que les miens sur des points de réflexion ou de comportement précis.

\section{Difficultés}

Il existe une incertitude fréquente concernant la capacité contenante du cadre judiciaire qui peut proposer peu de limites et des sursis multiples avec des décisions aléatoires, ce qui donne à certains moments un sentiment de lâchage du jeune. Certains sujets ont été en garde à vue dès l'âge de 9 ans puis de nombreuses fois sans rencontrer de butée judiciaire à leurs actes. En termes de structure psychique, afin de permettre l'apparition de la pensée chez certains adolescents, il aurait mieux valu commencer par un séjour en prison dans le quartier des mineurs avant l'admission au CER, c'est-à-dire d'abord contenir et empêcher que les tensions ne soient expulsées sous la forme d'un acte violent, puis écouter.

Par ailleurs, le cadre administratif du CER impose une contrainte temporelle importante: la durée de séjour est de cinq mois, mais elle peut, si nécessaire, passer à dix mois et plus.

\section{Caractéristiques de la population accueillie}

- Ces mineurs âgés de 13 à 17 ans et demi ont commis de multiples actes parfois très graves de délinquance et de violence, souvent avec des atteintes à des personnes dépositaires de l'autorité publique. Ils ont épuisé précédemment toutes les autres institutions éducatives, 
et les consultations pédopsychiatriques et psychologiques antérieures ont été inefficaces. Ils ne manifestent parfois aucun respect de la contrainte pénale, du contrôle judiciaire, et ne vont parfois pas aux rendez-vous donnés par le juge. Le CER est souvent leur dernière chance avant la prison ou pour éviter d'y retourner alors que certains y ont déjà fait des séjours de plusieurs mois.

- Leurs troubles sont le plus souvent la conséquence de négligences et de maltraitances précoces, de processus d'emprise (beaucoup d'adolescents déclarent vouloir habiter leur vie durant à côté de leurs parents pour les soigner et/ou leur acheter de la nourriture). Ils ont pu être exposés à des parents toxicomanes, paranoïaques, profondément dépressifs, délirants, souvent incapables de se décentrer d'eux-mêmes. $70 \%$ d'entre eux ont été exposés à des scènes de violences conjugales pendant les deux premières années de leur vie et certains y pensent encore tous les jours. Ainsi un jeune de 16 ans m'explique qu'il n'arrive pas à s'endormir parce que le soir, quand il n'a plus d'activité, surgit dans son esprit l'image de son père appuyant un couteau sur la gorge de sa mère après avoir fermé la porte de l'appartement à clef pour qu'elle ne puisse pas partir avec son enfant. Il avait alors trois ans. Pour trouver le sommeil, il faut qu'il téléphone à son amie dont la voix douce lui permet de «tirer un rideau» devant l'image angoissante. L'utilisation du téléphone portable étant interdite au CER, un autre dispositif sera mis en place. Si l'on ne tente pas de mettre en lien ce passé traumatique lointain et le présent, la difficulté à laquelle est confronté cet adolescent ne reste qu'un «problème d'endormissement».

- Ces traumatismes relationnels précoces, décrits par E. Bonneville (2015) et moi-même, ont des conséquences bien connues: atteinte de la pensée, attachement insécure désorganisé désorienté ou évitant avec un refus de dépendance, hyperactivité avec trouble attentionnel et impulsivité, violence sous différentes formes, troubles psychiatriques de type dépressif, tentatives de suicide, etc. À cela, il faut ajouter un éventuel facteur tempéramental, en particulier dans les troubles des conduites.

- $\quad 88 \%$ de ces jeunes sont des Maghrébins souvent de première génération, et il existe alors une dimension culturelle particulière (Berger, 2016). 85 \% des éducateurs sont eux aussi maghrébins, ce qui, dans cet établissement, a un effet de similitude positif ( $c f$. infra). 
Le traumatisme lié à l'immigration n'est pas un facteur causal: l'histoire des parents montre que la violence intrafamiliale éventuelle était déjà présente dans le pays d'origine.

- La précarité n'est pas non plus un facteur causal ici. Plus de $50 \%$ des parents travaillent. $30 \%$ des autres présentent des troubles psychiatriques qui nécessitent souvent que leur allocation d'adulte handicapé soit gérée par une tutelle.

\section{Points communs et différences entre les deux groupes}

Avant de travailler en CER, ma pratique clinique s'est déroulée pendant 35 ans dans un service de pédopsychiatrie comprenant deux hôpitaux de jour et une unité d'hospitalisation à temps complet dédiés en grande partie aux soins aux enfants très violents âgés de trois à douze ans, avec une prise en charge individuelle quotidienne, une temporalité thérapeutique possible sur plusieurs années, un placement familial spécialisé (ces enfants étaient tous placés hors de leur famille) et des visites médiatisées protectrices gérées par les soignants, activités couplées à un dispositif de recherche (Berger, 2008, 2012a, 2012b).

La violence de ces enfants étant elle aussi le plus souvent la conséquence de négligences et de maltraitances précoces, je pensais donc que la longue expérience thérapeutique acquise avec eux allait être assez rapidement utilisable pour les adolescents. Ce ne fut que très partiellement le cas.

En effet, les jeunes admis au CER sont différents des adolescents que nous suivions jusqu'à leur majorité après leur passage en hôpital de jour ou en hospitalisation complète pendant leur enfance: même lorsque ces derniers étaient en état de crise, ils gardaient la trace des moments de mentalisation partagés précédemment et, sur ce fond relationnel établi, on pouvait relativement rapidement donner sens aux difficultés présentes en les mettant en lien avec leur histoire. Au contraire, les adolescents admis en CER ont longtemps grandi sans protection réelle, sans évaluation de la manière dont leurs besoins fondamentaux étaient satisfaits ou non et sans placement stable, alors qu'à la lecture de leur dossier ou à l'écoute de leur histoire, cela paraissait indispensable. Et leurs difficultés à mentaliser et à nouer des liens étaient beaucoup plus importantes. On peut résumer ces différences dans le tableau suivant. 


\begin{tabular}{|c|c|}
\hline ENFANT DE MOINS DE 12 ANS & ADOLESCENT \\
\hline $\begin{array}{l}\text { Accepte la dépendance affective par } \\
\text { rapport aux soignants. }\end{array}$ & Présente une volonté d'indépendance. \\
\hline $\begin{array}{l}\text { Dépend totalement de l'adulte dans la } \\
\text { réalité }\end{array}$ & Dépend de l'argent des trafics illégaux. \\
\hline $\begin{array}{l}\text { Peut s'appuyer physiquement sur le corps } \\
\text { du professionnel référent (proximité, } \\
\text { câlin). }\end{array}$ & $\begin{array}{l}\text { S'appuie contre le corps du professionnel } \\
\text { (affrontement). }\end{array}$ \\
\hline $\begin{array}{l}\text { S'appuie sur la pensée de l'éducatrice/ } \\
\text { éducateur en prise en charge en hôpital } \\
\text { de jour. }\end{array}$ & $\begin{array}{l}\text { S'appuie sur le groupe extérieur (le } \\
\text { quartier). }\end{array}$ \\
\hline $\begin{array}{l}\text { Parle de ce qu'il ressent au professionnel } \\
\text { témoin impliqué. Affects de détresse } \\
\text { partagés pour la première fois. }\end{array}$ & $\begin{array}{l}\text { Parle difficilement de ses émotions, qu'il } \\
\text { a du mal à identifier. }\end{array}$ \\
\hline $\begin{array}{l}\text { Peut comparer de lui-même la relation } \\
\text { offerte par le professionnel référent avec } \\
\text { l'attitude parentale. }\end{array}$ & $\begin{array}{l}\text { Ne peut pas procéder à cette } \\
\text { comparaison. Psychisme envahi par la } \\
\text { groupalité familiale. }\end{array}$ \\
\hline $\begin{array}{l}\text { Accepte les mises en représentation, } \\
\text { récits à deux, dessins, jeux de rôle, etc. }\end{array}$ & N'accepte pas ces types de médiation. \\
\hline $\begin{array}{l}\text { Lors des contacts avec ses parents, peut } \\
\text { être protégé par des visites médiatisées. }\end{array}$ & $\begin{array}{l}\text { Capacité de fuguer pour aller dans sa } \\
\text { famille. }\end{array}$ \\
\hline $\begin{array}{l}\text { Visites médiatisées au cours desquelles } \\
\text { l'enfant parvient à différencier sa pensée } \\
\text { de celle de ses parents. } \\
\text { Possibilité de travailler sur les imagos } \\
\text { parentales même à l'adolescence. }\end{array}$ & $\begin{array}{l}\text { Incapacité des sujets de penser sur leurs } \\
\text { parents (imagos intouchables) et de } \\
\text { penser en présence de leurs parents. } \\
\text { Adolescent et parents doivent être } \\
\text { reçus séparément pendant longtemps } \\
\text { (cf. infra). }\end{array}$ \\
\hline $\begin{array}{l}\text { Peut s'étayer sur les pairs en groupe } \\
\text { pédagogique. }\end{array}$ & $\begin{array}{l}\text { Est envahi psychiquement et excité par le } \\
\text { groupe, donc nécessité d'une pédagogie } \\
\text { individuelle, ou honte du sujet face au } \\
\text { groupe s'il n'arrive pas à réaliser un } \\
\text { exercice. }\end{array}$ \\
\hline Force physique contenable. & $\begin{array}{l}\text { Grande force (lutte corps à corps avec les } \\
\text { éducateurs). }\end{array}$ \\
\hline $\begin{array}{l}\text { «Fugues» très rares. La tension est gérée } \\
\text { dans la relation. }\end{array}$ & Fugues fréquentes en cas de tension. \\
\hline Pas de consommation de drogue. & $\begin{array}{l}\text { Diverses addictions qui gênent la pensée } \\
\text { et l'apprentissage. }\end{array}$ \\
\hline
\end{tabular}


J'ai donc dû modifier considérablement mon mode de pensée et de travail pour m'occuper de ces adolescents, en prenant en compte les traces psychiques et corporelles laissées par les négligences et les maltraitances et le rôle du quartier.

\section{Quelques-unes des traces des traumatismes relationnels précoces}

\section{Les troubles du schéma corporel}

Chez les enfants âgés de 4 à 12 ans, grâce à des bilans psychomoteurs spécifiques (réalisés par Fabien Da Rosa), nous avons mis en évidence des troubles du schéma corporel habituellement peu explorés, comme l'impossibilité de ramper, de se retourner au sol, de marcher à quatre pattes - alors que ces acquisitions sont normalement réalisées à 10 mois -, une faible résistance à la poussée, une impossibilité de reconnaître les émotions sur le visage d'autrui, et d'autres difficultés. En grandissant, le sujet adolescent a fini par faire ces acquisitions, mais on sait que ces troubles ont pu exister et avoir eu un impact sur sa construction psychique. En effet, dans la toute petite enfance, on pense avec son corps, en appui sur les expériences corporelles.

Par ailleurs, des traces de ces difficultés anciennes persistent. Des parties du corps ne sont pas identifiées; par exemple un adolescent de 16 ans ne sait pas où se situent les sourcils, les tempes, la paume des mains sur son corps, et ce, sans qu'il s'agisse d'un problème de vocabulaire. Des difficultés de repérage dans l'espace peuvent être présentes: lors d'un bilan de psychomotricité, un jeune déclare pouvoir aller d'un bout à l'autre d'une grande pièce en 2 pas et fait plusieurs tentatives malgré leur échec prévisible. Et la trace des perturbations de l'image du corps explique probablement les nombreuses chutes que font ces adolescents lors des gestes de la vie courante: il est fréquent qu'ils soient plâtrés à la cheville, au poignet, etc., me donnant l'impression d'avoir une «consultation d'éclopés».

Le test de l'image du corps de Claire Meljac montre des distorsions importantes dans la représentation de l'image de soi. Beaucoup de jeunes âgés entre 15 et 17 ans échouent à construire de manière cohérente un visage ou un corps de profil à partir des pièces qui leur sont fournies les unes après les autres, sur un fond fixe représentant les cheveux et la nuque ( $c f$. infra). Cette épreuve est normalement réussie à 11 ans 11 mois. On peut se demander jusqu'à quel point ces sujets voient le visage de leur interlocuteur comme structuré, «unifié» et, sinon, de quelle manière cela intervient dans les relations qu'ils ont avec autrui. 
Test de l'image du corps de Claire Meljac (évocation) Monsieur A, 15 ans:

CORPS:

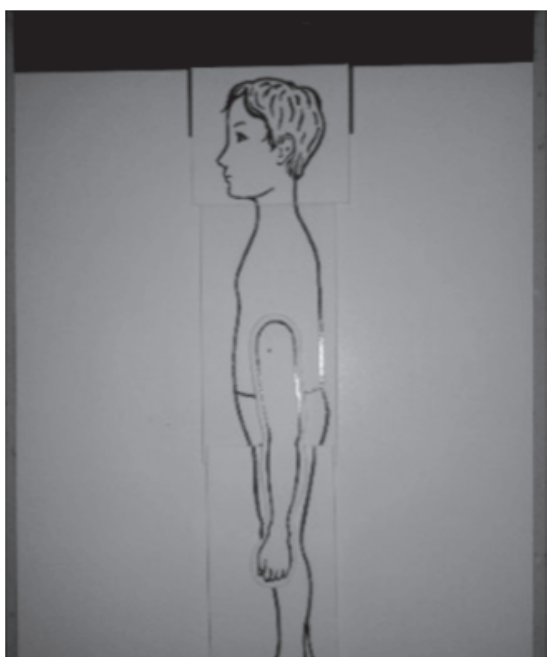

Monsieur C, 16 ans:

CORPS:

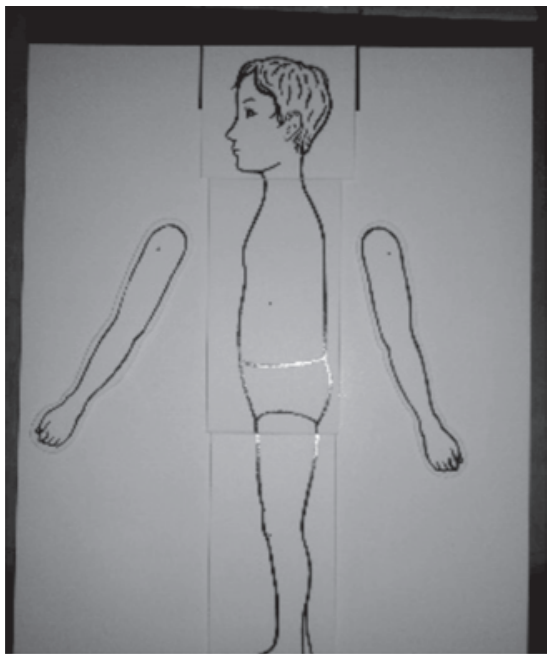

VISAGE:

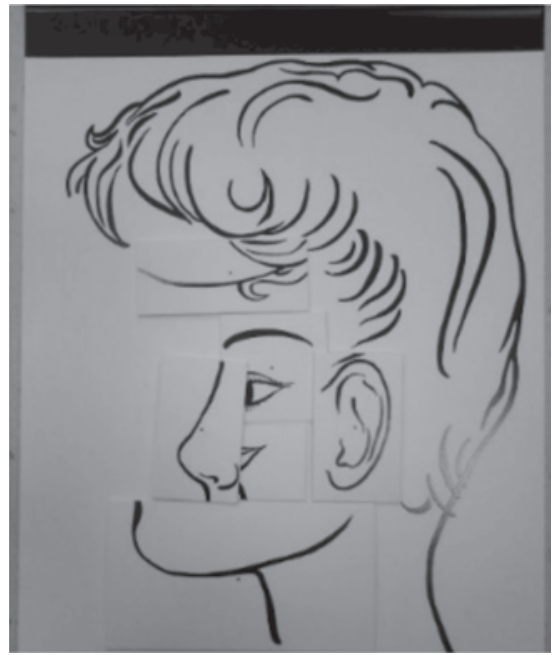

VISAGE:

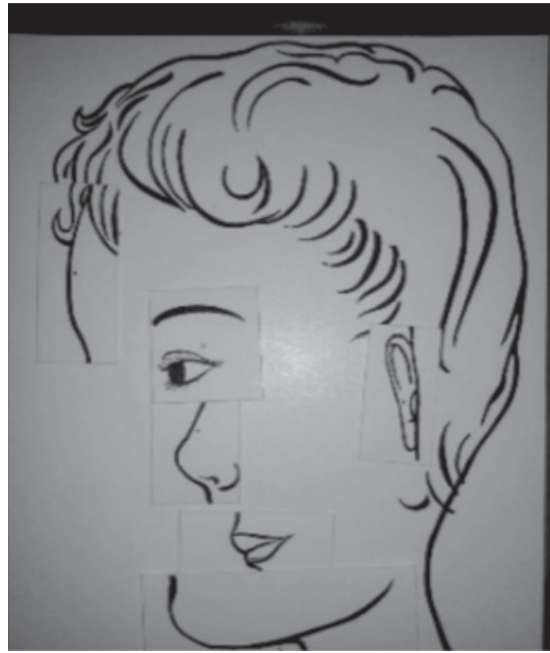

- En plus de l'hyperkinésie avec trouble attentionnel que présentent la plupart de ces adolescents, beaucoup d'entre eux ne sont jamais dans une position de détente: être assis, c'est «rouiller», disent-ils. 
Et le fait de ne jamais être dans une position de confort corporel participe à rendre impossible la scolarisation. Rappelons que nous sommes une société assise (Da Rosa). Par ailleurs, comme depuis leur petite enfance leur fond tonique n'est pas un tonus de repos mais une hypertonie, certains de ces sujets ne peuvent pas percevoir qu'ils «font une crise» parce qu'elle n'est pas ressentie par eux comme une variation importante de leur état tonique. C'est souvent l'environnement qui leur indique qu'il s'est produit en eux une manifestation tonico-émotionnelle de grande intensité.

- Enfin, ces adolescents sont incapables de garder une tension en eux: «quand je m'énerve, c'est trop tard» expliquent-ils. On essaye donc de repérer ensemble les signes corporels que chacun d'eux identifie comme des précurseurs de l'impulsivité: gorge sèche, tension musculaire, sensation de chaleur thoracique, etc., afin qu'ils puissent prendre aussitôt un médicament calmant d'action rapide avant de frapper sans retenue. Cette médication pourrait ainsi leur éviter de détruire les relations avec leur environnement et d'être renvoyés des stages de formation qu'on leur propose. Un problème groupal apparaît alors toutefois: "Dans le quartier, si je ne réagis pas immédiatement, je ne suis plus un "zeub” [pénis], les autres vont penser que je suis une "mouille" [terme élégant pour désigner une fille] et prendre la confiance. Je préfere faire une connerie et aller en prison que prendre un traitement», me déclare un jeune de 17 ans qui sort de 5 mois d'emprisonnement. Dans le mode de relation groupale établi, il faut être vigilant et toujours prêt à se montrer le plus fort: il faut donc sans arrêt se muscler, car la musculature actuelle n'est jamais considérée comme suffisante, nous y reviendrons. Et souvent, il faut avoir en permanence un couteau sur soi dès qu'on sort du CER, car «on ne sait jamais». Donc les médicaments qui diminuent l'impulsivité, ce cancer de la vie sociale, sont considérés comme féminisants et longtemps refusés pour cette raison.

\section{Les lacunes de la permanence de l'objet}

Une autre trace des négligences précoces est une acquisition incomplète de la permanence de l'objet. Ces sujets présentent donc souvent une grande sensibilité aux changements, qui les renvoie à l'imprévisibilité traumatique du monde de leur petite enfance. Ceci peut entraîner une réaction d'agressivité extrême face à un imprévu même minime, tel qu'un changement 
d'horaire, dans la même journée, d'une activité programmée. Connaître cette intolérance au changement aide les éducateurs à comprendre certaines colères disproportionnées et peut être mis en lien, lors d'un entretien ultérieur avec l'adolescent, avec les discontinuités émotionnelles et physiques qu'il a vécues dans le passé.

\section{L'atteinte cérébrale}

On pourrait enfin évoquer ici les conséquences de l'atteinte cérébrale liée au stress et d'autres facteurs importants.

\section{Le rôle du quartier}

\section{Fuir la maison, chercher la protection à l'extérieur}

Mohamed, 16 ans, admis au CER, m'explique que lorsqu'il avait cinq ans sa mère a fait une grave dépression. Il est alors placé dans une famille d'accueil où il se sent bien, puis à six ans, d'une manière incompréhensible pour lui, il est confié à son père, qui se révèle maltraitant: "Mon père se foutait de moi. Il ne passe pas par la parole, il frappe. Personne ne me protégeait, je sortais jusqu'à minuit et rejoignais les grands de 15 ans. Ils m'ont adopté». Il se sent donc accueilli, trouve là une sorte de deuxième famille, mais il est aussi rapidement intégré dans l'économie souterraine de ce groupe qui lui propose de livrer de la drogue. "À sept ans, je gagnais 200 à 300 euros par jour, plus que les salaires cumulés de mes deux parents». On mesure ici l'aspect «dérisoire» des propositions éducatives concernant des stages afin que Mohamed acquière un minimum de compétences professionnelles lui permettant ensuite de gagner sa vie en travaillant. Peut-on "remonétariser» la valeur de l'effort et les valeurs en général? Partie perdue d'avance? Pas sûr.

Quand j'interroge Mohamed sur ce qu'il faisait de cet argent, il me répond qu'il achetait sans arrêt des habits de marque et des Pokémons. Je lui dis simplement qu'il devait avoir beaucoup de choses. Non, m'explique-t-il, car il s'en débarrassait au fur et à mesure. Je lui demande s'il donnait ses vêtements et ses cartes à d'autres jeunes, et sa réponse, dite sur un ton neutre, un peu dépressif, est négative: il les jetait à la poubelle. On constate comment dans cette sorte de compulsion d'achats faciles, ce qui compte, c'est le moment où l'on prend possession, mais le plaisir qui s'en suit est de courte durée, de sorte qu'il faut acheter sans cesse d'autres habits. Ces biens ne sont pas offerts dans le cadre d'une relation à l'autre et dans un plaisir partagé.

C'est pourquoi il reste une petite chance de gagner la partie si un professionnel parvient à créer avec un tel adolescent une relation solide et 
confrontante ( $c f$. infra, la butée). Tous deux peuvent alors construire ensemble un projet, expérience que ce sujet n'a jamais vécue dans sa famille. Un projet pour soi, individualisé, avec l'idée de se projeter dans le futur, à la place du projet «tribal» du quartier qui est de vivre groupalement, éventuellement du trafic de drogues sous la menace permanente de rivaux et de la police. De plus, travailler donne au sujet de l'empowerment, c'est-à-dire le sentiment qu'il peut agir sur son sort. Beaucoup de ces adolescents disent qu'ils auraient voulu entrer en apprentissage à un âge plus jeune, en étant payés 300 à 400 euros par mois, ce qui leur aurait permis d'avoir un espace psychique personnel, différent de la filiation familiale et groupale. Mais perdre l'affiliation groupale entraîne de la culpabilité et l'abandon d'une partie de son identité (Lacroix et Gilbert, 2015). C'est ainsi que, peu à peu, grâce aux entretiens et au travail des éducateurs, les relations entre Mohamed et sa mère s'améliorent et cet adolescent décide de distendre les liens avec le groupe du quartier. Il m'explique avec ses mots: «Je veux travailler parce que j'ai envie de faire plaisir à mon entourage et à moi-même. Je veux me respecter [on ne l'a guère respecté à certains moments de son enfance]. J'ai connu le délire de l'adrénaline lors des cambriolages. Je veux connaître un autre délire, celui du travail». Mais le groupe ne le lâche pas comme ça: il lui est demandé de participer à un cambriolage. Il ne sait pas comment se dégager de ce lien qui a été essentiel pour lui, sans le rompre. Il trouve la solution suivante: il refuse de participer au vol, mais donne 50 euros à celui qui le sollicite pour montrer sa solidarité, sa "participation» à la vie groupale.

\section{L'intérieur plus angoissant que l'extérieur}

Revenons à la mère de Mohamed que je reçois en présence de son fils. Elle décrit sa dépression sévère: elle ne se lavait plus, se couchait la nuit tout habillée, restait allongée pendant la journée, et ne surveillait plus son fils qui a failli se noyer à cette époque. Suite à un signalement effectué par une enseignante d'école maternelle, un juge des enfants le place dans une famille d'accueil et ordonne à la mère de suivre des soins, ce qu'elle ne fait pas, car, me dit-elle, dans sa culture cela aurait signifié qu'elle était folle. Lorsque je lui demande si elle a des souvenirs d'elle enfant à l'âge où Mohamed partait de la maison la nuit, elle se lève et va au fond de la pièce en nous tournant le dos pour qu'on ne puisse pas voir ses émotions sur son visage. Je la reçois alors sans son fils, et elle m'explique que ses parents ne s'occupaient jamais d'elle, ils travaillaient tout le temps et la laissaient seule jusqu'à très tard le soir. Elle s'effondre en larmes en évoquant les moments où, âgée de cinq ans, debout face aux nombreuses clés accrochées 
au mur - son père était gardien d'immeubles -, elle se préparait à les essayer les unes après les autres jusqu'à trouver celle qui lui permettrait de partir dans la nuit afin de rencontrer des personnes inconnues qui se préoccuperaient d'elle. Il est possible que l'arrivée de Mohamed à l'âge de cinq ans ait réactivé la sensation d'effondrement qu'elle avait éprouvée au même âge. Il s'agit d'un processus classique au cours duquel l'enfant réel fait ressurgir chez le parent des émotions liées à sa propre enfance.

Beaucoup d'adolescents ont ainsi commencé à errer dès l'âge de cinq ans pour échapper à la violence terrifiante ou à la négligence parentale. Plus qu'une fugue du domicile, il s'agit de partir pour fuir un lieu où l'on ressent de la terreur (comme Mohamed avec son père) ou une sensation de solitude extrême (comme la mère de cet adolescent), et c'est alors un mode de survie. Et puis il y a aussi la phrase de Yazed Kherfi dans son livre Repris de justesse (2000): "Ma mère était "éteinte" jusqu'au décès de son mari [...]. Le sujet qu'on évitait toujours dans les discussions entre jeunes, c'était celui de nos parents et de nos familles; les copains étaient largement plus attirants que ce que l'on pouvait trouver dans nos maisons.»

\section{Le leurre: le quartier comme oripeau d'identité}

Oripeau: étoffe, broderie ou ornement de faux or ou de faux argent; faux éclat, apparence brillante et trompeuse (Petit Robert).

Les sujets violents ont une identité fragile, morcelée. Comment pourraient-ils se construire une identité solide quand le seul modèle dont ils disposent est un parent qui frappe ou qui est négligent ou très déprimé ou délirant, ou autre, quand la permanence de l'objet n'est pas suffisamment intégrée, quand aucun sourire ne vient refléter le sourire adressé à autrui, quand la représentation de son propre corps n'est pas stable, quand des réminiscences hallucinatoires du passé peuvent ressurgir le soir ou dans la journée? Et quand pour eux l'intérieur et la vie à la maison sont plus angoissants que le monde extérieur, contrairement à ce qui se passe pour un enfant qui vit dans un milieu familial sécurisant. Le sujet à l'identité incertaine cherche un miroir, de l'identique, pour savoir qui il est, comment il est, expérience qui lui a manqué dans sa petite enfance. Pour cela, il essaye de s'appuyer sur le moindre point de similitude visible, sur des points parcellaires tels que la couleur de la peau qui transforme en personne rassurante l'autre ressenti d'abord comme inquiétant; le statut d'immigré; le rituel collectif de l'affrontement physique; une identité de genre qui consiste pour les garçons à considérer et à traiter les filles comme inférieures; la structure 
spatiale du quartier considéré comme un territoire (dont il peut être difficile et angoissant de franchir les frontières); une théorie générale du complot qui «permet» de comprendre la société (le monde serait dirigé par les Juifs et les Illuminati), etc.

Un exemple: un jeune métis monte dans un tram en fumant. Une psychologue originaire de l'Inde, présente, lui demande gentiment d'arrêter, car cela gêne les passagers. Réponse immédiate: «tu es raciste». La psychologue lui fait remarquer qu'elle aussi a la peau foncée, et il répond «alors on est pareil» et arrête immédiatement de fumer. On comprend l'intérêt de la présence des éducateurs maghrébins, ce qui n'empêche pas les éducateurs non maghrébins d'effectuer un travail de grande qualité.

Le rituel du combat dans le code de l'honneur est une de ces modalités d'identité groupale. Hocine explique que dans son quartier, on se bagarrait très vite, pour divers problèmes. "Ça commence pour rien, on s'amuse et ça devient violent, ça bascule rapidement. Si tu ne refuses pas un tête-à-tête, c'est que tu as des couilles, même si tu te retrouves au sol; c'est si tu refuses que là, tu as honte. Les choses doivent se régler directement, de moi à lui.» Il faut se battre pour appartenir au groupe, être comme les autres.

La parole ne sert pas à négocier, mais participe à l'augmentation de la tension et remplace la parole familiale dont le rôle aurait pu être de penser ensemble les divergences. Cette difficulté d'utiliser la fonction apaisante des mots s'est parfois, là aussi, "structurée» dès la petite enfance. Hocine déclare: "À la maison, ce n'était jamais calme. J'avais peur quand j'étais petit, mon père me frappait fort et frappait aussi ma mère. J'ai pris des coups pour rien, j'avais mal, j'avais besoin d'être consolé, ma mère ne le faisait pas, je ne sais pas pourquoi. Parfois je me sens devenir comme mon père, ça vient tout seul. Mais je sais me contrôler: dans ces cas, je tape les murs. J'ai des moments tristes quand je me demande pourquoi mon père tapait pour rien. Lui poser la question ne changera rien. » Ainsi, aucun recours aux mots n'est possible, pas de phrases de consolation de la part de la mère, questionner est voué à l'échec. Hocine est au bord des larmes au cours de notre entretien, lorsqu'il commence à penser à ce qui lui a manqué.

D'où, comme on le dit souvent, l'importance de séjours hors du quartier suffisamment longs pour "désintoxiquer» le sujet de ces codes.

Comme la famille ne permet pas à ces jeunes de se forger une identité reposant sur une image globale d'eux-mêmes et sur des bases sécurisantes, ils se construisent alors tant bien que mal à partir de bribes d'identité parcellaires et complètement extérieures, qu'ils empruntent au quartier et 
à la rue, comme s'ils se vêtaient de bouts de vêtements mal taillés et faits de bric et de broc. Mais toutes ces tentatives ne permettent de se constituer qu'une identité reposant sur des apparences, et morcelée en réalité.

Dans certaines situations, c'est au modèle extérieur du djihadisme que le sujet fera appel pour se sentir avoir une identité moins fragile.

On sait maintenant que les jeunes djihadistes peuvent présenter des profils très variés. Mais un profil particulier concerne la première vague d'attentats en France. Les médias ont insisté sur l'itinéraire commun des tueurs à partir de l'adolescence - délinquance, prison -, mais on est passé très vite sur ce qui s'est passé auparavant dans leur petite enfance. Or il existe un point commun: Mohamed Merah (le tueur de Toulouse), Medhi Nemmouche (le tueur du musée juif de Bruxelles), les frères Kouachi (les tueurs de Charlie Hebdo), etc., ont tous été placés en institution pendant leur adolescence et même plus tôt pour certains parce qu'ils vivaient dans un milieu familial fortement négligent et maltraitant. Mohamed Merah, par exemple, a été exposé à des scènes de violences conjugales très fortes, et il a fugué dans la rue vers l'âge de quatre ans. Il évoquait «un homme qui parlait dans sa tête», a subi la grande violence de son frère dont le pitbull déchirait ses cahiers et l'a mordu à plusieurs reprises. Mohamed n'a été placé qu'à quatorze ans.

Et les modèles les plus attractifs, séducteurs mêmes, sont ceux porteurs de certitudes concernant le «bien et le mal». Pierre Lévy-Soussan fait remarquer que la dénomination «État islamique» propose une organisation, un «État», et aussi une fonction paternelle chez des sujets qui ont eu des pères principalement absents ou violents.

\section{Quelle modalité relationnelle proposer à ces sujets, quand on est psychanalyste?}

Il ne s'agit pas ici de traiter ou de réparer, mais de permettre au sujet d'effectuer un travail de réflexion sur lui, afin d'atténuer les traces des négligences et maltraitances et de faire contrepoids à la partie désorganisatrice des identifications groupales et familiales.

\section{Les modèles cliniques habituels pour permettre un travail psychique}

André Carel (2016) propose quatre modèles. 


\section{1-1. Le modèle contenant de l'autorité}

Le cadre est ici presque suffisant à l'intérieur de l'établissement - «presque», car pour certains jeunes il me semblerait préférable que l'établissement soit fermé.

\section{1-2. Le modèle du jeu}

Il est impossible à utiliser ici: tout est pris au sens littéral; le mot égale la chose. «Nique ta mère» n'est pas seulement une insulte à la famille donc une atteinte narcissique identitaire, l'expression est prise au sens sexuel littéral, d'où une réponse systématiquement explosive. Ces sujets n'ont aucun humour et pensent être l'objet de moquerie si on essaye de plaisanter avec eux. Ils ne plaisantent d'ailleurs pas non plus entre eux. Ils ne jouent pas avec les pensées, les mots, mais seulement en actes. En particulier, le jeu $\mathrm{du}$ «pas vu pas pris» lors des cambriolages leur procure un plaisir addictif, et plusieurs jeunes m'ont expliqué voler sans nécessité et seulement pour l'excitation que cela leur procure. Autre jeu, celui de détruire en vrai, la destructivité réelle. Après les émeutes de 2005 dans les banlieues «sensibles», j'ai reçu en expertise plusieurs émeutiers mineurs qui m’ont dit la même chose que ce qu'ils déclarent souvent aux magistrats devant lesquels ils sont convoqués: s'ils ont brûlé ou saccagé des classes maternelles, une bibliothèque, ce n'était pas parce qu'ils étaient révoltés contre le système scolaire, contre la société, mais c'était "pour s'amuser». En tout cas, c'était ainsi à cette époque encore antérieure au communautarisme. Un jeu qui peut être à haut risque, car des personnes extérieures à ces actions ont été grièvement brûlées dans ces incendies.

Arrêtons-nous sur l'expression «pour s'amuser». Ces sujets ne peuvent pas jouer à la bagarre, c'est-à-dire faire semblant de se taper, car ils n'ont pas acquis le "comme si», n'ont pas pu se construire d'aire transitionnelle qui permette de penser que c'est à la fois du vrai et de l'imaginaire. Leur seul jeu est fréquemment l'échange de coups, sorte d'érotisme musculaire. Quand j'explore ce qui leur fait plaisir dans la vie, leur seule réponse, accompagnée d'un grand sourire, est souvent: «j'aime la bagarre, quand d'autres se battent, je vais m'y mêler, même si je n'ai rien à y voir». Et quand je leur demande de me parler de leurs premières «bêtises », ils prennent le temps de réfléchir, se souviennent avec un sourire ému de leurs débuts de délinquant, comme si c'était en quelque sorte leurs premiers pas dans la société. 


\section{1-3. Le modèle du rêve, du fantasme}

Il est impossible à utiliser pour presque tous. Ils n'ont aucun espace interne de rêverie, ne comprennent pas le mot «rêvasser ». Je demande à l'un d'eux: «Peux-tu imaginer ce que tu ferais si tu gagnais au loto?» Réponse: «Je n'y réfléchirai que quand j'aurai les millions devant moi, sinon ça ne sert à rien d'y penser.» Ou la «rêverie» du sujet est «formatée», sans scénario fantasmatique: "Mon rêve, ce serait de vivre avec plein de meufs, de voitures de sport avec beaucoup d'argent», c'est-à-dire comme certains joueurs de football professionnels. Un éducateur et moi proposons à un jeune une tentative d'entrer dans une aire de rêverie en débutant devant lui un psychodrame sur un thème banal, ce que nous avons envie de faire comme activité en fonction du temps, avec l'espoir qu'il va se joindre à nous alors qu'il a refusé de participer au début. Il a alors une forte réaction de panique: «Vous êtes fous! Ce n'est pas vrai ce que vous dites, arrêtez!». Et pour de tels sujets, leur univers interne est menaçant car il est littéral, non différencié de la réalité externe. Ils ne peuvent donc pas laisser «flotter leur pensée» en rêvassant.

Avec un autre adolescent qui n'arrive pas à s'endormir, j'essaie d'évoquer un moment de rêverie en lui demandant: «Est-ce que tu penses que ta mère te lisait des histoires ou te chantait une chansonnette le soir?» Sur un ton sarcastique, il me répond que je dis des «bêtises». Puis, sur un ton ironique, il me demande: «Et vous, on vous chantonnait une chanson le soir?» Ma réponse est: «Je pense que oui». Quelques secondes de silence, et j'ajoute: «Et ce devait être doux». Le visage de ce jeune particulièrement endurci se transforme alors pendant un dixième de seconde lorsque j'énonce ma deuxième partie de phrase, comme s'il était traversé par un sentiment profond et peut-être douloureux. Pourquoi cette réponse de ma part? Il s'agit d'une réponse métaphorique, car comme beaucoup, je ne me rappelle pas les berceuses qu'on me chantait quand j'étais enfant. Mais ce jeune très violent et tendu refuse la moindre exploration de son intériorité, cela lui est insupportable, et les entretiens ne peuvent progresser que si j'accepte de montrer préalablement une partie - modérée - de mon intériorité. C'est lui qui, quelques jours après cet entretien, demandera à me rencontrer à nouveau "pour continuer la discussion », en précisant que je suis bizarre. Un certain désir d'explorer des chemins bizarres est apparu en lui.

$\mathrm{Ne}$ pas pouvoir faire semblant signifie ne pas pouvoir fantasmer, ne pas pouvoir construire de scénario, ne pas pouvoir utiliser le dessin spontané, ni entrer dans un récit à deux, sorte de squiggle parlé, chacun proposant une 
phrase qui s'articule avec la précédente. Donc pour ces sujets qui n’ont pas d'espace intrapsychique, le modèle de la psychothérapie est non seulement inadapté, mais il risque en plus d'être persécutant car le sujet ressent vite ce cadre comme étant dans un registre dominant-dominé, d'où l'intérêt de la présence de l'éducateur qui triangule la relation entre le psychiatre ou le psychologue et l'adolescent.

\section{1-4. Le modèle du soin}

Ces adolescents n'ont aucune idée de ce qu'est un lien au sens où nous l'entendons; ils ne connaissent que des liens d'emprise, de soumission, des liens claniques, des liens qui asservissent, ou des liens qui lâchent. Ou encore le lien groupal créé par l'affrontement physique, qui est une des formes de la destructivité que j'évoquerai plus loin: il faut se battre avec l'autre pour être pote, c'est-à-dire survivre à un combat réel. «Ce n'est pas important si tu es battu, l'important c'est que tu acceptes le combat». Donc ces sujets n'ont pas de représentation d'un lien d'attachement sécurisant et de ce que peut être un care giver.

\section{Pourquoi ne pas penser?}

Ces sujets ont de bonnes raisons de ne pas penser sur le passé et sur le présent. Leur question : «ça sert à quoi de penser?». Il ne sert à rien de tenter d'y répondre.

Pourquoi ne pas penser? Pour ces sujets, le risque permanent est que reviennent à leur esprit des affects terribles, pensons à la mère de Mohamed qui s'effondre en se rappelant sa détresse à l'âge de cinq ans devant les trousseaux de clés. "De quoi vous vous mêlez, vous grattez là où ça fait mal», me déclare un jeune après des questions anodines. Puis «Ce qui se passe dans ma famille ne vous regarde pas ", alors que je n'ai pas proposé de rencontrer ses parents. On peut aussi penser que certains adolescents ont honte de leurs pères et mères qu'ils ne trouvent pas présentables et ne veulent donc pas qu'on les rencontre. Mais ceci entrave le travail sur les imagos parentales, en particulier sur la manière dont peut surgir chez de tels sujets la dimension mélancolique de leurs parents qu’ils ont intériorisée. «Penser, ça fout les morts ", me dit un adolescent.

Penser, c'est risquer de mesurer l'étendue du désastre. La lucidité a un prix lourd: ces jeunes sont souvent à la limite des larmes en ma présence lorsqu'ils parviennent à évoquer leur passé. Et ceci même lorsqu'ils vont mieux. Un journaliste souhaite rencontrer des enfants pris en charge 
dans notre service hospitalier et devenus adultes, pour parler avec eux de la manière dont ils ont vécu les soins. Les deux jeunes auxquels je téléphone pour leur proposer de rencontrer ce journaliste réfléchissent pendant une semaine, puis me font une réponse négative car ils craignent que les émotions du passé ne ressurgissent en eux et ne les replongent dans leur état antérieur. Car il existe 3 sortes de souvenirs:

- Les bons souvenirs, agréables à évoquer, encore faut-il qu'il y en ait.

- Les mauvais souvenirs évocables, qui concernent des images désagréables mais supportables.

- Les mauvais souvenirs non évocables car ils feraient ressurgir les émotions angoissantes du passé telles quelles, à l'état brut. Le passé est évoqué comme s'il était présent. C'est une des caractéristiques du traumatisme psychique: le temps se fige au moment où il se produit. Et c'est ce que redoutent ces adolescents.

Nous ferions peut-être comme eux étant donné ce qu'ils ont subi. Mais cela ne leur facilite pas la vie et les amène à ne penser que dans l'immédiat et sur l'immédiat. Et surtout, ne pas penser laisse encourir le risque de faire de nouvelles victimes.

On comprend que le mot que l'on entend le plus souvent est «normal», qui a plusieurs sens:

- L'impossibilité de penser que le monde puisse être autrement que la manière dont on l'imagine ou que les échanges avec autrui puissent se dérouler différemment de la manière dont ils se sont produits;

- La peur d'être anormal, de reconnaître l'existence d'une partie folle ou incontrôlable en soi;

- La peur d'être différent des autres membres du groupe;

- L'incapacité de se sentir, de s'entendre, de se voir, de conflictualiser en soi ce qu'on ressent (absence de fonction réflexive).

C'est pourquoi, comme l'a écrit Michael Balint, à une question, on n'obtient souvent qu'une réponse. Face à de telles réticences à penser, le risque est que le professionnel se retire de la scène, qu'il lève le camp et que l'adolescent se retire dans le sien.

\section{Alors comment faire émerger de la pensée?}

Comment obtenir de la substance psychique utilisable? 


\section{3-1. II faut tout d'abord sécuriser l'adolescent}

Le sécuriser par la présence de l'éducateur référent pendant tout l'entretien, car pour lui je suis un visage inconnu, donc menaçant. Dans ce contexte, l'éducateur, bien connu de lui, est une figure d'attachement sécurisante, base de sécurité préalable à l'exploration du monde, en l'occurrence ici à l'exploration de sa propre vie psychique, de ses sentiments. Habituellement, dans un travail psychothérapique dit classique, le fond sécurisant est constitué par le cadre, son rythme régulier, le matériel identique d'une fois sur l'autre (la table, les chaises). Et la personne même du psychothérapeute est sécurisante, il est à la fois une figure d'attachement parentale par sa présence régulière et calme, et celui qui amène hors des chemins connus. Avec les adolescents concernés, penser est tellement angoissant qu'on est obligé de faire appel à deux personnes différentes pour remplir ces deux fonctions.

De plus, si je réussis à éveiller un affect, l'adolescent n'est pas lâché après l'entretien avec les sensations corporelles et les émotions que cela a pu déclencher en lui: il fait ensuite un trajet en voiture avec l'éducateur, et tous deux peuvent reprendre ce qui s'est dit. La représentation que le jeune a de l'éducateur change car ce professionnel a vu et entendu le jeune être touché, donc l'adolescent sait que l'éducateur le voit différemment, et l'éducateur sait que le jeune le voit différemment. Cette présence de l'éducateur au moment de l'apparition d'un affect qui n'est plus vécu dans la solitude crée un lien différent: "il me suit partout depuis l'entretien", déclare un éducateur. Ce petit changement, n'est-ce pas ce qu'on peut espérer de mieux? Au cours de ces entretiens, l'éducateur, même s'il n'a pas pris la parole, devient central, sinon, s'il n'est pas présent, ces professionnels déclarent: "On récupère des cocottes-minute après l'entretien, on est le réceptacle de l'explosion.»

Autrefois, pour les enfants de moins de 12 ans en hôpital de jour, les entretiens individuels étaient confiés aux éducateurs (trices) ou aux infirmiers (ères) formés et supervisés, car ils assuraient une fréquence quotidienne et ils étaient présents au moment où un affect surgissait chez l'enfant. Sinon, si on dit à un tel enfant, lorsqu'il se sent mal ou devient violent, «tu en parles dans quatre jours au moment de ta séance de psychothérapie», la possibilité de comprendre ce qui a perturbé son état émotionnel a de fortes chances d'être perdue. Il faut au contraire une attention et une exploration immédiates du mal-être. Comme l'a montré le tableau de comparaison cidessus, ce dispositif n'est pas envisageable avec un adolescent non pris en charge précédemment. Cependant, le principe de contenance et de tentative 
de compréhension rapide par la personne qui est «bien au courant» de l'histoire et de l'état du sujet est valable dans les deux contextes.

Une autre dimension de la sécurisation concerne le lieu. Les entretiens se déroulent dans une Maison de quartier, sorte de Centre social dont une grande salle nous est louée au moment des rendez-vous. Il y a là un baby-foot, une table de ping-pong, une chaîne hi-fi avec de grands hautparleurs, etc., et nous devons chaque fois installer au centre une vieille table en formica autour de laquelle nous allons discuter. Ainsi les jeunes sont dans un cadre proche de celui qu'ils connaissent. Je ne suis pas certain qu'ils parviendraient à la même relative liberté d'expression s'ils se sentaient être sur mon territoire, c'est-à-dire si je les recevais dans mon bureau de consultation. À ce propos, comme l'indique Albert Ciccone, l'école de sociologie de Chicago a montré que si un psychologue blanc évalue le niveau intellectuel d'un enfant noir dans son bureau de blanc, il obtient un résultat bien inférieur à celui obtenu par un psychologue noir évaluant le même enfant dans son quartier ou sur son trottoir.

Sur ce fond ainsi sécurisé, peut-on faire émerger de la pensée? Dit autrement, ces sujets font l'expérience de pouvoir faire confiance aux éducateurs pour trouver un lieu d'apprentissage, pour contenir physiquement les conflits, et parfois les écouter lorsqu'ils se sentent mal, mais ils ne vont que rarement jusqu'à se confier sur leurs sentiments ou sur leur passé difficile. Il y a une différence entre faire confiance et se confier, ce qui ici consiste à explorer des parties douloureuses de son monde interne. Se confier signifie se confier à autrui, s'en remettre à, se reposer sur: il y a un certain abandon de soi livré aux bons soins de l'autre, comme lorsqu'on livre son corps à un médecin ou chirurgien.

\section{2-2. Reconnaître la méfiance du sujet}

Pour que le sujet puisse se confier, il faut d'abord souligner sa crainte. Je demande donc toujours au début de l'entretien quel est mon numéro de dossard: combien de psychologues ou de psychiatres l'adolescent a-til rencontrés auparavant. Cela varie de 7 à 17. Et cela lui a-t-il été utile? Réponse habituelle: «ça ne sert à rien ». J'introduis ainsi le fait que j'accepte la méfiance du jeune à mon égard.

Méfiance aussi, car, pour ces sujets, rencontrer un psychiatre signifie être fou. «Je ne suis pas fou», déclarent-ils d'emblée. Ou pour se moquer de moi : «je vais me suicider» avec un sourire, puis «c'est le genre de choses que vous attendez que je vous dise». D'où l'importance que l'entretien soit porté 
par l'éducateur, qui explique à l'adolescent avoir besoin de mon point de vue pour mieux comprendre comment s'occuper de lui dans la vie quotidienne. De plus, l'éducateur va pouvoir évoquer les actes dont le jeune ne parle pas spontanément soit par évitement, soit à cause d'un clivage.

Il faut souligner ici un processus qui survient fréquemment tant au niveau des parents que des adolescents, et que Freud a nommé «réaction thérapeutique négative »: si le sujet se laisse aller à montrer des sentiments, lors de l'entretien suivant il se referme sur son système de non-communication et peut même nier avoir tenu certains propos si on se permet de les évoquer. Le progrès semble effacé. Le sujet a pu avoir le sentiment de s'être trop découvert, et la méfiance reprend sa fonction protectrice; ou il est trop douloureux pour lui de constater qu'il a pu recevoir du bon, car ce n'est pas de la bonne personne qu'il l'a reçu, c'est avec ses parents qu'il aurait dû pouvoir partager ce qu'il ressentait, etc. Un professionnel qui n'est pas au courant de ce processus le prend pour une résistance et se décourage en pensant que le chemin accompli précédemment est annulé, alors que la réflexion pourra reprendre plus tard. Ainsi Ahmed, 16 ans, accepte de réaliser un récit à deux avec moi, chacun ajoutant une phrase à celle proposée par l'autre, le tout étant écrit au fur et à mesure par moi. Dans cette histoire, un garçon trouve des champignons comestibles dans la forêt, les rapporte à la maison, et les prépare pour le repas du soir. Ahmed: «Tout à coup la porte s'ouvre, c'est le papa qui rentre et l'enfant est content». Moi: «Le père goûte le plat, dit que c'est délicieux après toute une journée de travail, et félicite son fils d'avoir été capable de faire cela». Ahmed: «Merci papa, ça me touche beaucoup». Dans la réalité, ce père prête peu attention à son fils et ne tient aucune des promesses qu'il lui fait. Le lendemain, cet adolescent déclare aux éducateurs que j'ai critiqué son père dans ce récit, alors qu'il s'était au contraire permis d'imaginer un moment de plaisir partagé avec lui où chacun était heureux de la manière d'être de l'autre.

\section{Le modèle proposé: le jeu de la destructivité et le facteur $\mathrm{K}$ de Bion}

\section{Le jeu de la destructivité}

Ce terme, qui fait parfois l'objet d'une généralisation abusive, correspond à un processus psychique décrit par Winnicott (1975).

De nombreux travaux de psychologie expérimentale montrent que pendant les premiers mois de sa vie, l'enfant ne fait pas la différence entre le monde extérieur et lui-même. Lorsqu'il tient une balle, il ne perçoit pas la 
limite entre sa main et l'objet. Ou s'il a faim, un bébé ne perçoit pas où se situe l'origine de la douleur, c'est-à-dire si elle provient de l'intérieur de son corps ou de l'extérieur, ou si l'origine d'une action se situe en lui ou chez l'autre. Chez les sujets négligés ou maltraités, on peut retrouver plus tard les traces de cette indifférenciation entre soi et le monde extérieur. Ainsi un enfant violent âgé de 7 ans tient fortement le poignet de son éducatrice en lui disant «lâche-moi!». Ou un préadolescent dit à un infirmier qui le contient dans ses bras au moment d'une crise de violence: «je préfère être enfermé dans ma chambre car quand tu me tiens comme ça, je ne sens plus la limite entre mon corps et le tien et ça m’angoisse».

Puis, vers l'âge de quatre ou cinq mois, Winnicott décrit l'apparition du comportement suivant chez les nourrissons. Sans ambiguité, le bébé se met à exercer des gestes «cruels » sur ses proches, tire leurs cheveux, griffe, déchire, mord la tétine de son biberon ou autrui, avec un air qui ne laisse pas de doute concernant son intention de détruire. La réponse de l'environnement est alors fondamentale.

Si le parent «survit» sans représailles ni rétorsions, en retirant par exemple sa main mordue tout en donnant à l'enfant un autre objet à mordre; ou s'il transforme l'activité de morsure en un jeu, ou s'il arrête les gestes destructeurs et propose une autre occupation, l'enfant constate que son désir de détruire l'autre ne se réalise pas. Ce n'est qu'une pensée, sans destruction réelle. Il n'est donc pas tout puissant sur le monde; le «jeu de la destructivité» réalisé avec les parents lui permet de constater que la réalité est «indépendante» de lui, ses attaques en pensée n'ont pas d'effet sur la réalité, et il peut jouer sans risque avec ses idées, elles ne se réaliseront pas. Dit autrement, ce ne sont que des fantasmes. Un parent réel est donc un parent qui a résisté à de telles attaques sans se montrer trop atteint ou agressif en miroir, et son état ne dépend pas des pensées de l'enfant.

Or justement, les jeunes dont il est question ici n'ont jamais pu faire cette expérience, parce que leurs parents étaient absents physiquement ou psychiquement et donc indifférents et sans réaction «vivante»; ou parce qu’ils se montraient trop atteints par les attaques de l'enfant et se déprimaient alors; ou parce qu'ils exerçaient des représailles selon une loi du talion: "Tu me mords avec ta force, je te mords (ou je te tape) avec ma force» sans faire jouer la différence de maturité entre eux et l'enfant. L'enfant considère alors que sa pensée est effectivement capable de détruire l'autre, ou qu'elle a un effet tel qu'elle rend l'autre destructeur. La différence entre fantasme et réalité ne s'établit pas complètement. L'autre n'est donc pas complètement réel. 
Revenons à la clinique des adolescents violents. Beaucoup d'entre eux ont besoin de passer par une épreuve d'affrontement physique avec leur éducateur référent, accompagnée d'une contention au sol, pour que ce professionnel commence à être investi comme une personne sur laquelle il pourra s'appuyer. Et c'est une des dimensions du rituel de la bagarre: on n'a un lien réel avec l'autre que si les deux protagonistes ont survécu à un tel affrontement. Les adolescents qui refusent - sagement - ce rituel sont transparents aux yeux des membres du groupe de leur quartier.

Et il en est de même lors des moments de joute verbale dans les entretiens avec moi: l'échange ne vise pas alors en premier à obtenir des informations, à faire penser, encore que cela se produise souvent grâce au facteur $\mathrm{K}$, mais à ce qu'ait lieu un d'échange durable avec un affrontement sans rupture, ce qui ne peut survenir que si le professionnel répond en son nom propre: une attaque personnelle nécessite une réponse personnelle impliquant de la consistance, sans chercher à prendre le dessus. Au début, le but de l'échange, c'est donc l'échange lui-même.

Allons encore plus loin. Lors des entretiens, quand je pose la question «As-tu pu dire une fois à ton père que tu n'étais pas d'accord avec lui?», ces jeunes m'indiquent que ma question est littéralement inimaginable, aussi incongrue que si j'avais demandé «Penses-tu que la terre est plate?» Puisque ces pères sont souvent très violents, leur dire qu'on pense différemment d'eux serait suicidaire. Le père peut en outre être abandonnant, et lui faire des reproches entraînerait encore plus d'absence de sa part à titre de représailles. On constate comment toute expérience de destructivité a été impossible à expérimenter antérieurement, alors que c'est elle ainsi que la résistance de l'objet et son absence de rétorsion qui créent l'aire du fantasme.

On peut aussi remarquer que ces jeunes peuvent frapper leurs mères si elles essaient de leur interdire de sortir le soir ou de fumer du haschich à la maison (et il faudrait protéger certaines de ces femmes par des interdictions de retour au domicile imposées à l'adolescent). Pourtant, leurs mères ne sont pas critiquables pour eux, quelles qu'aient pu être leurs négligences et leur absence d'empathie ou de protection.

En l'absence d'expérience possible de destructivité, le parent n'est pas réellement inscrit comme objet de pensée dans la psyché, et aucune élaboration n'est réalisable concernant les images parentales, qui demeurent intouchables. C'est tout un pan de l'activité psychique qui n'a pas pu se développer. Or, le seul lien utilisable qui permet de construire sa vie psychique et de supporter l'absence est un lien en pensée. 
Il en est de même dans le fonctionnement groupal clanique: on peut s'entretuer lorsqu'il existe un différend à propos d'argent, mais il règne une omerta psychique, c'est-à-dire qu'on ne se permet pas de critiquer l'autre, en premier lieu le patriarche, encore moins devant autrui.

\section{Le facteur K de Bion (1962)}

Le psychanalyste Bion décrit trois éléments constitutifs de la personnalité dès la petite enfance, les facteurs L (love, amour), $\mathrm{H}$ (hate, haïne), et $\mathrm{K}$ (knowledge, connaissance). Les deux premiers correspondent à nos mouvements pulsionnels. «K signifie la volonté de savoir sans insister sur la connaissance en elle-même», «ce n'est pas une chose que nous avons». K, c'est le besoin, le désir, et le plaisir de comprendre, d'apprendre de l'expérience.

La traduction clinique de cette théorisation m'est apparue lorsqu'au cours d'expertises judiciaires, je faisais passer le test de Brunet Lézine à des bébés âgés de neuf mois, donc en pleine période d'angoisse de l'étranger. Face à mon visage qui leur est inconnu, ces bébés manifestent leur méfiance en fronçant les sourcils, puis la passation débute avec ses petites épreuves extraordinaires: prendre des cubes dans chaque main, retrouver un cube sous un morceau de tissu, sortir le rond de la planchette, tout ceci étant totalement nouveau pour le nourrisson. Dès que s'effectuent les premières découvertes communes, l'angoisse disparaît, un lien s'établit. Ce n'est pas un lien d'amour, ou de confiance, mais un lien profond de plaisir cognitif partagé. Le nourrisson attend clairement et avec une certaine patience l'épreuve suivante. Je ne souris pas lorsque nous découvrons quelque chose ensemble, car cela pourrait signifier que c'est «ma satisfaction» et le sujet pourrait s'en sentir dépossédé, mais je manifeste une jubilation discrète: "ouuuuuuui». Ce plaisir cognitif, non conflictuel et non sexualisé, est neutre par rapport aux affects classiques, et c'est aussi pour cela qu'il soude les équipes. C'est le plaisir d'articuler sa pensée avec celle de l'autre, de féconder mutuellement nos esprits pour aboutir à une création nouvelle; c'est une des formes de la mutualité.

Le but est donc d'amener l'adolescent à une découverte commune, une «co-créativité», par une curiosité saine. «Explique-moi? Je ne comprends pas, aide-moi à comprendre. Attends, tu vas trop vite.» «Tu m'as appris des choses lors du dernier entretien», apports concernant la vie psychique en général dont je donne quelques exemples à partir de la rencontre précédente, etc. Il faut essayer d'être un objet naiff ignorant, donc non intrusif, et qui cherche à apprendre/comprendre le monde. Et à faire émerger un point 
inconnu jusqu'alors de nous deux, ce qui va créer un sentiment de surprise accepté puisque c'est le sujet qui en est en partie à l'origine.

Un exemple: Fabio, 14 ans, se présente comme très imbu de lui-même, toujours habillé élégamment, les cheveux gominés. Il m’explique "faire tomber» une multitude de filles, il envisage de travailler plus tard dans un grand restaurant, bref, il ne laisse aucun espace pour questionner une éventuelle souffrance psychique. Je lui dis alors: «C'est curieux, tu m'expliques à quel point tout marche bien dans ta vie, mais je me demande s'il n'y a pas un œil très sévère en toi qui t'oblige à être le meilleur, sinon il te dit que tu ne vaux rien. Si c'était vrai, alors tu aurais en toi en permanence un regard exigeant. Et ce serait fatiguant. Que penses-tu de cette idée? Tu peux me dire que je me trompe, je ne suis pas susceptible.» Cette remarque porte sur les défenses narcissiques, être le meilleur ou n'être rien. Fabio ne répond rien sur le moment. Mais sa curiosité est à l'œuvre puisqu'il demande à me revoir plus tôt que prévu et m'interroge dès le début de l'entretien suivant: «Dites, ce regard que j'ai dans la tête, il vient d'où?» La curiosité est partagée, nous pouvons réfléchir ensemble.

Et ce n'est parfois qu'à la fin de la rencontre que les défenses s'atténuent, lorsque l'adolescent sait qu'il va quitter la pièce. Ainsi Karim fait preuve d'une opposition totale pendant l'entretien. Il explique être venu seulement parce que le juge a décidé une obligation de soin. J'accepte son refus et parle de lui avec son éducatrice. Mais après avoir annoncé la fin de l'entretien, je fais deux interventions. Comme il revient sur l'inutilité de notre discussion, je lui propose l'hypothèse suivante: quand il était petit, il n’a jamais pu faire l'expérience que dire ses soucis à quelqu'un pouvait l'aider. Surprise, il répond: «Je suis d'accord.» Il ajoute que dire ses soucis ne sert à rien. J'émets alors une deuxième hypothèse: quand il émettait des signaux, pleurs, paroles, air triste ou autre, cela tombait dans le vide (sa mère a fait une grave dépression suite au décès de son mari lorsque Karim avait 15 mois). Deuxième surprise, iI répond: «c'est fort possible». Silence. Je ne dis rien. Il demande: "Alors c'est quand le prochain rendez-vous puisqu'il y a une obligation de soins?» C'est donc au moment où le jeune ne se sent plus en situation de contrainte relationnelle que certains éléments importants peuvent apparaître, d'où l'importance de ne pas regagner le vestiaire trop vite.

Un autre domaine relativement neutre de curiosité partagée consiste à évoquer l'histoire et la géographie. On peut questionner le sujet sur le lieu d'où sa famille est originaire, souvent un pays étranger, et sur ce qu'il 
pourrait nous en apprendre lors de l'entretien suivant après qu'il se soit renseigné, habituellement sur internet. Cette démarche peut être d'autant plus intéressante que, le plus souvent, ces jeunes refusent au départ de répondre à la moindre interrogation sur leur histoire personnelle et familiale. Or un minimum de représentation de ses origines est indispensable à tout progrès psychique. Cette curiosité quant à l'origine, source de découverte en commun, constitue aussi un ciment intéressant de la relation (cf. l'utilisation du génogramme par S. Gilbert, 2016). On constate donc que, souvent, ce n'est pas par une réflexion directe sur les agir que l'on va progresser, même si cela devra être abordé à un moment ultérieur.

\section{Conclusion}

On pourrait dire que le trajet psychique «idéal» consisterait à passer du besoin de similitude à l'étayage, puis à la co-création, et au plaisir d'agir pour soi dans un projet et non pour survivre. Ce faisant, des processus s'intriquent qui concernent plusieurs niveaux: le corps propre du sujet, le corps familial, le groupe de pairs, la sensorialité et la représentation du monde du bébé, les réactions liées à l'adolescence. L'infans, celui qui n’a pas la parole, n'est ici pas seulement le bébé, mais aussi l'adolescent violent et hors la loi.

Et finalement, la partie infantile du sujet, avec sa capacité de réfléchir sur soi, est peut-être celle qui apparaît le moins spontanément et que nous allons tenter de faire émerger, car c'est sur elle que nous pouvons le plus nous appuyer.

Maurice Berger

drmberger@laposte.net

\section{Références}

Berger, M. (2008). Voulons-nous des enfants barbares? Paris: Dunod.

Berger, M. (2012a). Les visites médiatisées: fondements théoriques et cliniques. Dans Les visites médiatisées dans le cadre de la protection de l'enfance (p. 61-95). Paris: Éditions GREUPP.

Berger, M. (2012b). Soigner les enfants violents. Paris: Dunod.

Berger, M. (2016). De l'incivilité au terrorisme. Comprendre la violence sans l'excuser. Paris: Dunod.

Bion, W. R. (1962). Learning from experience. Londres: Karnac Books.

Bonneville, E. (2015). Les traumatismes relationnels précoces. Toulouse: Éditions Eres.

Carel, A. (2016). Liens premiers, violences, analyse du Surmoi idéal. Dans Violences dans la parentalité (p. 29-39). Paris: Dunod. 
Gilbert, S. (2016), De la rupture à la quête: processus de "liaison» inhérents au génogramme libre. Colloque international Enfance, adolescence, famille et vulnérabilités: enjeux psychopathologiques, perspectives thérapeutiques, éducatives et sociales. Université de Caen Normandie, 12 octobre.

Kherfi, Y. (2000). Repris de justesse. Paris: Éditions La découverte.

Lacroix, O., et Gilbert, S. (2015). Se désaffilier pour mieux s'affilier. Criminologie, 48 (2), 167-187.

Meljac, C., et Fauconnier, E., et Scalabrini, J. (2010). Épreuve de schéma corporel révisée. Paris: Les Éditions du centre de psychologie appliquée.

Winnicott, D. W. (1975). L'utilisation de l'objet. Dans Jeu et réalité (p. 120-131). Paris: Gallimard. 